\title{
Hashimoto Thyroiditis: Diffuse Vs Nodular? A Case Study In A Rural Tertiary Care Hospital
}

\author{
Volga Harikrishnan, Sudha.V*, Janice Alphonsa and Chitra Srinivasan
}

Department of Pathology, Saveetha Medical College, Saveetha Nagar, Thandalam, Chennai, Tamilnadu, India.

\section{ABSTRACT}

Background: Hashimoto thyroiditis is an autoimmune disease caused by production of autoantibodies against thyroglobulin and thyroid peroxidase enzyme. Prevalence is $2 \%$ in general population and incidence is seven times more in females between 30-50 years of age. It may present as euthyroid, hypothyroid or hyperthyroid state. There is an increased frequency of Hashimoto's thyroiditis (HT) and HT associated thyroid cancer. HT can be presented as diffuse or nodular lesion. The aim of this study is mainly to find out the incidence of diffuse and nodular lesions in HT in patients who came for FNAC for thyroid enlargement.

Methods: Retrospective analysis of Hashimoto thyroiditis cases diagnosed by FNAC over the period of six months in Department of Pathology. Review of the clinical records of patients for further clinical, biochemical and radiological details to find out incidence of diffuse and nodular HT.

Result: In this study there is increased incidence of HT.Both types (Diffuse and nodular) were present. Mostly occur at the age group of 20 to 50 years and commonly seen in females. Diffuse type had all type of hormonal status (Hyper/Hypo/Euthyroidism) but all the cases of nodular type had only hypothyroid status. Clinical presentation of nodular lesions confirmed with sonographic findings.

Conclusion: The rate of occurrence of nodular lesion in Hashimoto thyroiditis with hypothyroidism is high among females aged 20-40 years when compared to diffuse lesions.

Keywords: Thyroiditis, Diffuse, Nodular, Hypothyroidism.

\section{Introduction}

Hashimoto thyroiditis is an autoimmune disease caused by production of autoantibodies against thyroglobulin and thyroid peroxidase enzyme. Prevalence is 1 to $4 \%$ in general population and incidence is seven times more in females between $30-50$ years of age. ${ }^{[1]}$ It may present as euthyroid, hypothyroid or hyperthyroid state. Initial mild hypothyroid symptoms seen which gets severe on progression of disease. Hashimoto thyroiditis usually detected by ultrasound guided FNA. This technique is beneficial because it helps to select patient who need surgery and to avoid surgery in unnecessary patient who have non neoplastic disease

Hallmark feature is lymphocyte infiltration into thyroid follicle. It causes significant morbidity. Hashimoto thyroiditis is clinically presented as diffuse or nodular lesion. Diffuse lesion is more commonly encountered in clinical practice and it may lead to hypothyroidism, detected by ultrasound.

Diffuse lesion is enlargement of entire gland without producing nodularity. Nodular lesion is where the thyroid gland enlargement produce nodularity. Nodular Hashimoto thyroiditis may predispose to development of thyroid carcinoma so close monitoring of Hashimoto thyroiditis for any neoplastic change is required. However nodular lesion is not common, recently its occurrence is on the raise.

This study is mainly to find out the incidence of diffuse and nodular lesions in patients who came for FNAC for thyroid enlargement and to correlate those features with other clinical and pathologic parameters.

\section{Materials and Methods}

This is a retrospective study. In this study all cases diagnosed as Hashimoto thyroiditis or lymphocyte thyroiditis by FNAC, over the period of six months (From July 2015-December 2015) in department of Pathology in our Institution were included. Other thyroid lesions were excluded from this study. Nodular and diffuse lesions are usually identified by clinical examination and ultrasonogram. Clinical (Age and sex of the patient, duration of symptoms, clinical presentation and hormonal status by using thyroid function test reports) and cytological (Hurthle cell changes, lymphocytic infiltration and association of malignancy) correlation was carried out.

\section{Result}

This retrospective study was done in Pathology department of Saveetha Medical College with a sample size of 105. All 
these 105 patients have been diagnosed of some thyroid disorder and were subjected to FNAC procedure. And the following results were obtained.

It was found that the thyroid lesions mostly occur at the age group of 20 to 40 years as shown in Chart 1 . Females $(70 \%)$ were more common than the males $(30 \%)$.

According the FNAC procedure done in 105 population with thyroid lesions diagnosed by FNAC. The following results were obtained.18 cases were diagnosed to have Hashimoto thyroiditis(17.14\%).57 and 29 cases were diagnosed to have Colloid goiter(54.28\%) and Nodular hyperplasia(27.62\%) respectively. Only one case of Follicular neoplasm $(0.95 \%)$

Out of the 18 patients of HT most of them had (11patients) diffuse enlargement of thyroid region and 7 had nodular lesions. Most of them had this enlargement for duration of
2-7 years. Majorityof them are under age group of 20-40 years of age. Mostly lesions are prevalent among females (16 cases) $93 \%$ and only two cases were found to be in males $6.2 \%$ as shown in Chart 3 .

We retrospectively analysed the ultrasonogram(USG) findings in cases diagnosed as HT by FNA procedure. USG was done in all the cases. 11(61.11\%) cases showed diffuse enlargement and 7 with multiple nodules or with single nodule which were also found by clinical examination $(38.88 \%)$ (Chart 4). Ultrasound image of nodular lesion shown in Fig 1:

Details regarding thyroid hormone status of patients with HT shown in Table 1

Irrespective of types (diffuse and nodular lesion) all the cases showed the features typical to that of HT as shown in Fig 2.

\section{TABLE 1:}

\begin{tabular}{|l|c|c|c|}
\hline Hashimoto thyroiditis type of lesion & Euthyroid status & Hyperthyroid status & Hypothyroid status \\
\hline Diffuse type (11) & $1(9.1 \%)$ & $4(36.4 \%)$ & $6(54.5 \%)$ \\
\hline Nodular type (7) & 0 & 0 & $7(100 \%)$ \\
\hline
\end{tabular}

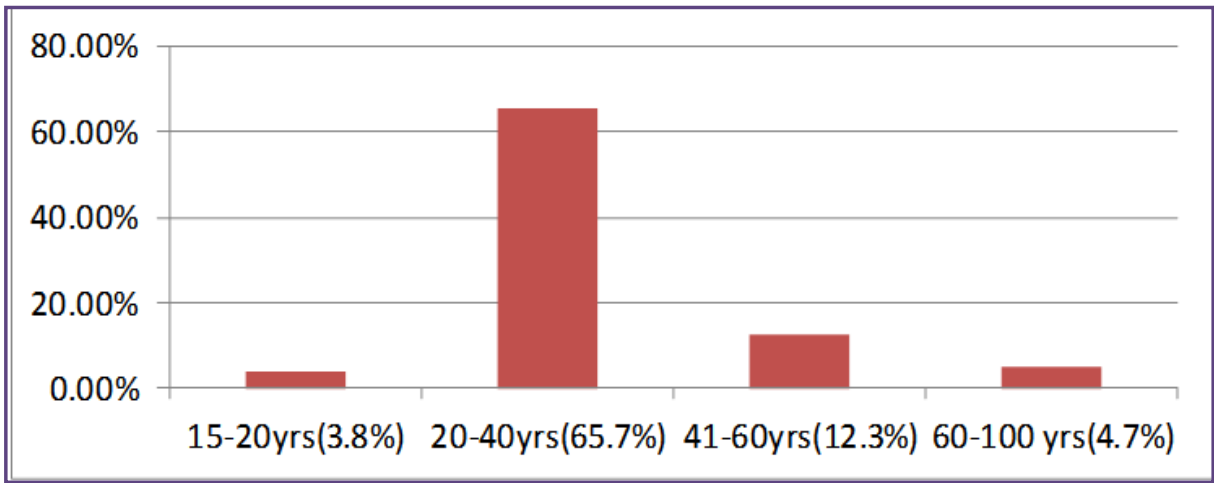

CHART 1: AGE DISTRIBUTION IN THYROID LESIONS:

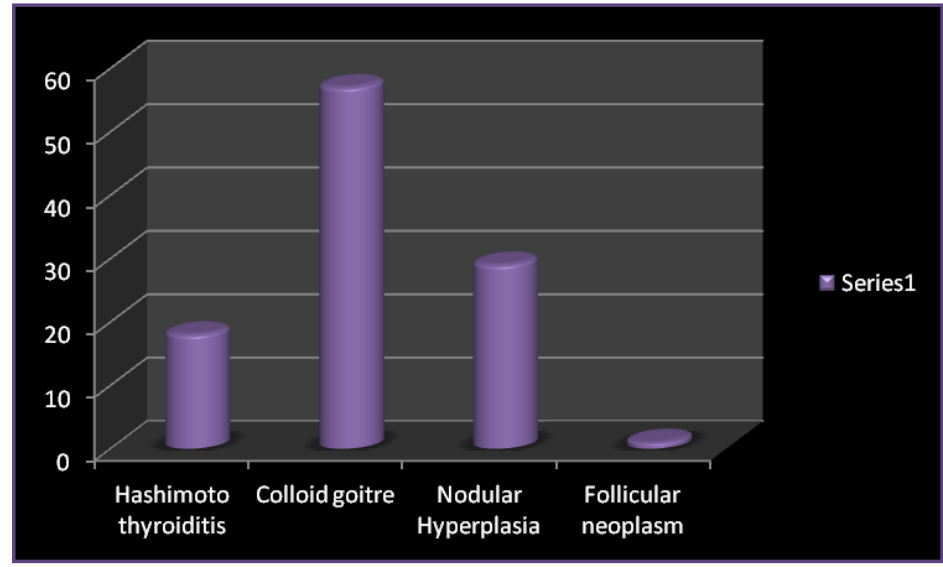

CHART 2: DISEASE DISTRIBUTION: 


\section{Sex distribution}

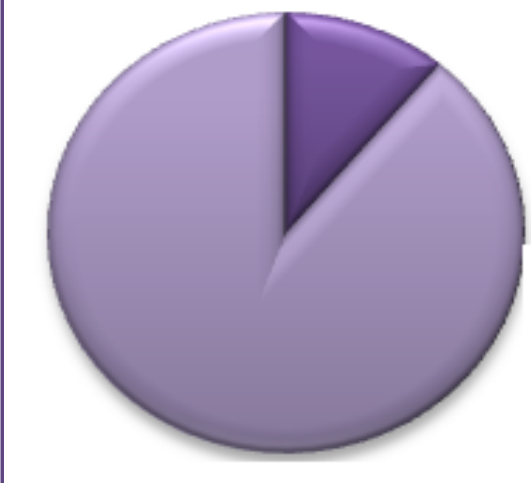

Male

Female

\section{CHART 3: SEX DISTRIBUTION IN HT:}

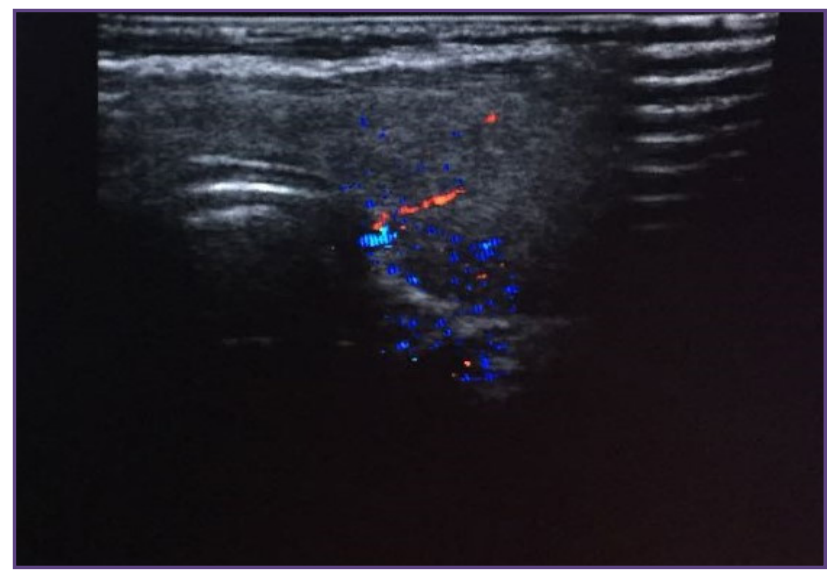

Fig. 1: Ultrasound image of HT-Thyroid with nodularity and increased vascularity within the nodule.

\section{Dicussion}

Hashimoto thyroiditis (HT) is one of the common autoimmune conditions encountered in clinical practice these days. Hashimoto thyroiditis is caused due to production of auto antibodies to thyroperoxidase enzyme; thymoglobulin and TSH receptors. The prevalence of HT is 1 to $4 \%$. ${ }^{[1]}$ In our study it was more than this (17\%).

HT can present as diffuse or nodular lesion. In Diffuse type there is entire gland enlargement with no nodularity, Nodular lesions have many nodularity or single nodule seen.

${ }^{[2]}$ Nodular type is more endangering because in course of time it can lead to thyroid carcinoma. ${ }^{[3]}$ The present study showed no cases associated with any carcinomas.

HT was diagnosed based on clinical, radiological (ultrasonogram) and biochemical data or combination of all

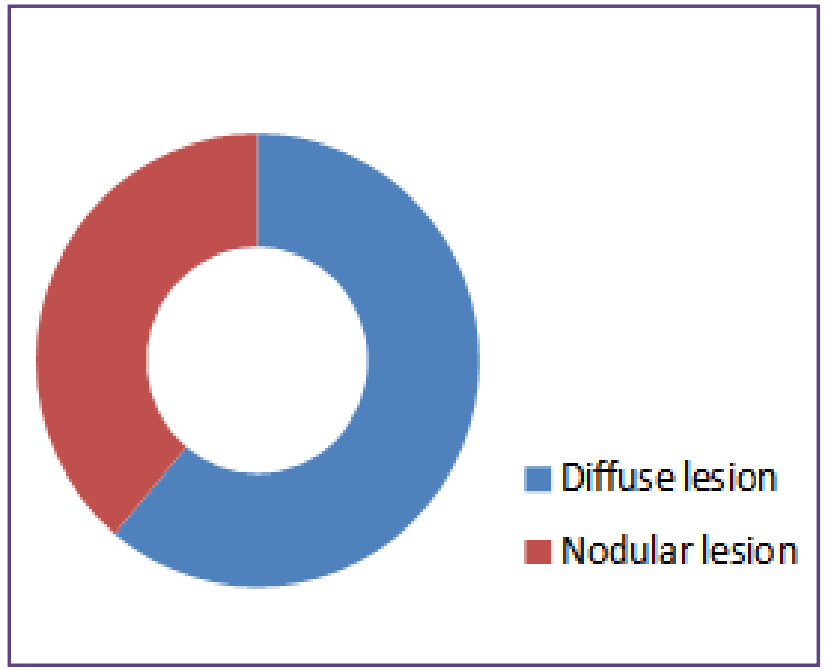

CHART 4: DIFFUSE VS NODULAR LESION:

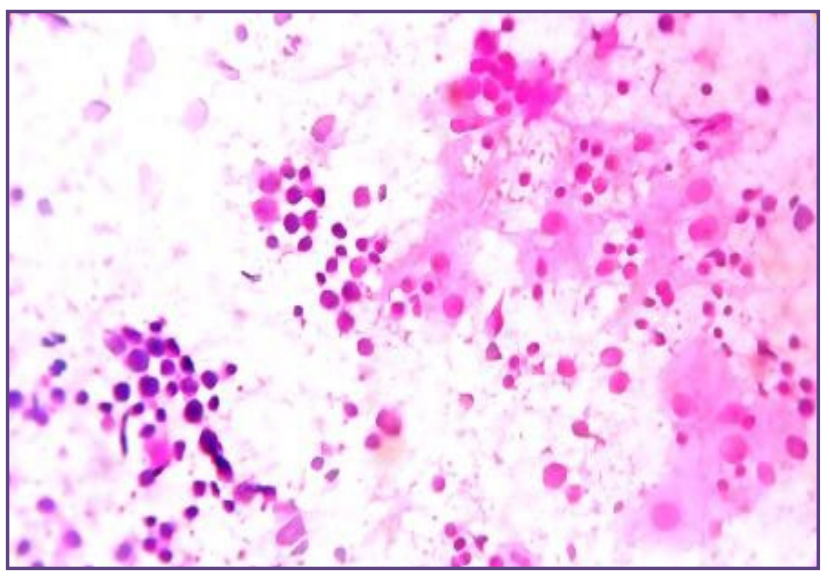

Fig. 2: H\&E (40X) FNAC smear showing thyroid follicular cells with Hurthle cell change and follicularlysis by the lymphocytes with varying stages.

features. ${ }^{[4]}$ Clinical details consisted of palpation of a firm, diffuse and rubbery thyroid. In our study there were diffuse (61.11\%) and nodular lesion (38.88\%) while palpating.

Biochemical data consisted of euthyroidism, hypothyroidism and hyperthyroidism. Hypothyroidism will have increased TSH along with subnormal T4 or FT4. Hyperthyroidism characterized by subnormal T4 or FT4 and normal TSH. But presence of euthyroidism did not exclude HT with associated other parameters to diagnose HT like cytological features and radiological features. Many studies showed that the most common cause of hypothyroidism is HT. ${ }^{[5]}$ Our study also proved the same with \% of diffuse HT and \% of nodular HT had hypothyroidism.

Ultrasonogram was usually performed to detect the typical HT pattern to measure thyroid size and to find out 
the presence of thyroid nodules. ${ }^{[6]}$ Two categories were considered: the "goitrous, regardless of nodules" and the "nodular, regardless of goiter". In our study the clinical presentation of diffuse Vs nodular lesions further confirmed by noticing the ultrasound image findinds. The results were similar as mentioned above.

FNAC is considered more cost-effective, simple and superior in diagnosing HT than antibody screening. ${ }^{[7]}$ Hashimoto thyroiditis can also be diagnosed by using fine needle aspiration technique guided by ultra-sonogram. ${ }^{[8][9]}$ The FNAC findings in case of HT is infiltration of thyroid gland with lymphocytes, B cells and T cells. ${ }^{[10]}$ and high cell yield consisting of Hurthle cells which have abundant eosinophilic granular cytoplasm with delicate vascular channels running through these cells. These cells were arranged in crowded three-dimensional aggregates, sheets, and in microfollicular pattern.

The clinical complications of hashimoto thyroiditis include, dermatomyositis, gottren sign(The lesions begin as non-palpable flat macules or patches $)^{[11]}$ and erythema with enlargement of thyroid gland. Presence of thyroid disease may progress to lymphoma. ${ }^{[12]}$

The present study is to find out the incidence of nodular lesions in present clinical scenario. Diffuse type of lesion is more commonly encountered however now nodular lesion is also on the rise. The reason to diagnose and treat nodular lesion is very important as it can cause severe fatal conditions like thyroid carcinoma.

\section{Conclusion}

From this study we conclude that rate of occurrenceof nodular lesion of Hashimoto thyroiditis with hypothyroidism was high among females aged 20-40 years when compared to diffuse type of HT. Appropriate measures for early diagnosis and treatment of nodular lesion must be undertaken so that progression of nodular lesion to malignancy can be reduced. Our study aims to suggest that nodular lesion is also on raise and so due attention and care must be given to treat these lesions

\section{References}

1. Gayathri BN, Kalyani R, Kumar ML, Prasad K. Fine needle aspiration cytology of Hashimoto's thyroiditis - A diagnostic pitfall with review of literature, Journal of Cytology. 2011 Oct-Dec; 28(4): 210-213.

2. Takashima S, Matsuzuka F, Nagareda T. Thyroid Nodule Associated With Hashimoto Thyroiditis: Assesement With US. Radiology.1992 Oct: 185(1):125-30

3. Paparodis R, Imam S, Todorova-Koteva K et al.Hashimoto's Thyroiditis Pathology and Risk for Thyroid Cancer. Thyroid. 2014 Jul 1; 24(7): 1107-1114.

4. Latina A, Gullo D, Trimarchi F et al. Hashimoto's Thyroiditis: Similar and Dissimilar Characteristics in Neighboring Areas. Possible Implications for the Epidemiology of Thyroid Cancer. PLOS ONE, 2013 Mar; 8(3):e55450.

5. Sood N, Nigam JS. Correlation of Fine Needle Aspiration Cytology Findings with Thyroid Function Test in Cases of Lymphocytic Thyroiditis. Journal of Thyroid Research, 2014 Apr; (2014), Article ID 430510.

6. Marcocci C, Vitti P, Cetani F. Thyroid Ultrasonography Helps to Identify Patients with Diffuse Lymphocytic Thyroiditis Who Are Prone to Develop Hypothyroidism. The Journal of Clinical Endocrinology and Metabolism. 1991 Jan; 72(1):209-13

7. Agrawal PC, Naik R, Panda KM. Diagnostic Role of Fine Needle Aspiration Cytology in Thyroiditis along with Thyroid Hormone Assay. Annals of Applied Bio-Sciences, 2016; 3(1).

8. Zhang JW, Chen ZJ, Gopinathan A. Focal Nodular Hashimoto's Thyroiditis: Comparison of Ultrasonographic Features with Malignant and Other Benign Nodules. 2016 Aug; 45(8):357-63.

9. Anderson L, Middleton WD, Teefey SA et al . Hashimoto thyroiditis: Part 2, sonographic analysis of benign and malignant nodules in patients with diffuse Hashimoto thyroiditis. $2010 \mathrm{Jul}$; 195(1):216-22.

10. Zeppa P, Cozzolino I, Peluso AL, Troncone G et al. Cytologic, flow cytometry, and molecular assessment of lymphoid infiltrate in fine-needle cytology samples of Hashimoto thyroiditis. Cancer.2009 Jun 25; 117(3):174-84

11. Matayoshi T, Omi T, Mayumi N, Kawana S. Hashimoto's thyroiditis with clinical manifestations resembling dermatomyositis: a case report. The Journal of Nippon Medical School. 2014; 81(2):106-9.

12. Chiang B, Cheng S, Seow CJ.Commonly forgotten complication of Hashimoto's thyroiditis.BMJ Case Reports. 2016 Oct $26 ; 2016$.

*Corresponding author:

Dr. Sudha. V, 8/90, Second Street,Krishna Nagar, Virugambakkam, Chennai-92, Tamilnadu, India.

Email: Sudh7828@gmail.com

Financial or other Competing Interests: None. 